Acta Crystallographica Section D

Biological

Crystallography

ISSN 0907-4449

\section{Structure of mouse IP-10, a chemokine}

\section{Talat Jabeen,‡ Philip Leonard, $\$$ Haryati Jamaluddin $\uparrow$ and K. Ravi Acharya*}

Department of Biology and Biochemistry, University of Bath, Claverton Down, Bath BA2 7AY, England

\footnotetext{
\# Present address: School of Biosciences, University of Birmingham, Edgbaston, Birmingham B15 2TT, England.

$\S$ Present address: Sareum Ltd, 2 Iconix Park, Pampisford, Cambridge CB22 3EG, England. - Present address: Faculty of Biosciences and Engineering, Universiti Teknologi Malaysia, 81310 Skudai, Johor, Malaysia.
}

Correspondence e-mail: bsskra@bath.ac.uk
Interferon- $\gamma$-inducible protein (IP-10) belongs to the CXC class of chemokines and plays a significant role in the pathophysiology of various immune and inflammatory responses. It is also a potent angiostatic factor with antifibrotic properties. The biological activities of IP-10 are exerted by interactions with the G-protein-coupled receptor CXCR3 expressed on Th1 lymphocytes. IP-10 thus forms an attractive target for structure-based rational drug design of antiinflammatory molecules. The crystal structure of mouse IP-10 has been determined and reveals a novel tetrameric association. In the tetramer, two conventional CXC chemokine dimers are associated through their $\mathrm{N}$-terminal regions to form a 12-stranded elongated $\beta$-sheet of $\sim 90 \AA$ in length. This association differs significantly from the previously studied tetramers of human IP-10, platelet factor 4 and neutrophilactivating peptide-2. In addition, heparin- and receptorbinding residues were mapped on the surface of IP-10 tetramer. Two heparin-binding sites were observed on the surface and were present at the interface of each of the two $\beta$-sheet dimers. The structure supports the formation of higher order oligomers of IP-10, as observed in recent in vivo studies with mouse IP-10, which will have functional relevance.

\section{Introduction}

Chemokines are involved in chemotaxis and activation of leukocytes in immune and inflammatory responses by interacting with their specific G-protein-coupled receptors (Moser \& Loetscher, 2001) and have been divided into C, CC, CXC and $\mathrm{CX}_{3} \mathrm{C}$ subfamilies on the basis of their $\mathrm{N}$-terminal cysteine positions (Zlotnik \& Yoshie, 2000; Ottonello, 2006).

Interferon- $\gamma$-inducible protein (IP-10; CXCL10; $10 \mathrm{kDa}$ ) belongs to the CXC family of chemokines and is secreted by a variety of cell types (Baggiolini et al., 1997). IP-10 acts as an immunoinflammatory mediator in Th1-type inflammatory diseases (Papadakis et al., 2004), rheumatoid arthritis (Ruschpler et al., 2003), cardiac allograft rejection (Zhao et al., 2002), multiple sclerosis (Sorensen, 2004), atherosclerosis (Heller et al., 2006), sarcoidosis (Sugiyama et al., 2006) and prostate cancer (Nagpal et al., 2006). The receptor for IP-10 has been recognized as CXCR3 (Loetscher et al., 1996), which is predominantly expressed on activated T lymphocytes (Th1; Sallusto et al., 1998) in addition to other cell types including NK cells, dendritic cells, macrophages and B cells (Loetscher et al., 1998; Qin et al., 1998). Two other physiological ligands for CXCR3 are monokine induced by interferon- $\gamma$ (Mig; CXCL9; Loetscher et al., 1996) and interferon-inducible T-cell $\alpha$ chemoattractant (I-TAC; CXCL11; Cole et al., 1998).
Received 16 October 2007

Accepted 13 March 2008

PDB Reference: mouse IP-10, 2r3z, r2r3zsf. 
Table 1

X-ray data-collection, processing and refinement statistics.

Values in parentheses are for the highest resolution shell.

\begin{tabular}{|c|c|}
\hline PDB code & $2 \mathrm{r} 3 \mathrm{z}$ \\
\hline Space group & $C 2$ \\
\hline Unit-cell parameters $\left(\AA{ }^{\circ}{ }^{\circ}\right)$ & $\begin{array}{l}a=109.9, b=71.5, \\
\quad c=39.6, \beta=110.0\end{array}$ \\
\hline Resolution range $(\AA)$ & $50.0-2.50(2.57-2.50)$ \\
\hline Total No. of measured reflections & 25840 \\
\hline No. of unique reflections & 9824 \\
\hline Completeness (\%) & $98.2(89.7)$ \\
\hline$V_{\mathrm{M}}\left(\AA^{3} \mathrm{Da}^{-1}\right)$ & 2.1 \\
\hline No. of molecules in the ASU & 4 \\
\hline$R_{\text {merge }}^{\dagger}(\%)$ & $9.1(30.4)$ \\
\hline$I / \sigma(I)$ & $6.9(3.2)$ \\
\hline$R_{\text {cryst }} \neq(\%)$ & 27.6 \\
\hline$R_{\text {free }} \S(\%)$ & 30.3 \\
\hline No. of protein atoms & 2044 \\
\hline No. of water molecules & 81 \\
\hline \multicolumn{2}{|l|}{ R.m.s. deviations } \\
\hline Bond lengths $(\AA)$ & 0.010 \\
\hline Bond angles $\left({ }^{\circ}\right)$ & 1.4 \\
\hline \multicolumn{2}{|l|}{ Mean $B$ factors $\left(\AA^{2}\right)$} \\
\hline Main-chain atoms & 55.5 \\
\hline Side-chain atoms and waters & 56.2 \\
\hline All atoms & 56.9 \\
\hline \multicolumn{2}{|l|}{ Ramachandran plot } \\
\hline Residues in most favoured regions (\%) & 77.8 \\
\hline Residues in additionally allowed regions (\%) & 19.6 \\
\hline Residues in generously allowed regions (\%) & 2.6 \\
\hline
\end{tabular}

$\dagger R_{\text {merge }}=\sum_{h k l} \sum_{i}\left|I_{i}(h k l)-\langle I(h k l)\rangle\right| / \sum_{h k l} \sum_{i} I_{i}(h k l)$, where $I_{i}(h k l)$ is the observed intensity of reflection $i$ and $\langle I(h k l)\rangle$ is the average intensity of multiple observations. $\ddagger R_{\text {cryst }}=\sum|| F_{\mathrm{o}}|-| F_{\mathrm{c}}|| / \sum\left|F_{\mathrm{o}}\right|$, where $F_{\mathrm{o}}$ and $F_{\mathrm{c}}$ are the observed and calculated structure-factor amplitudes, respectively. $\S R_{\text {free }}$ is equal to $R_{\text {cryst }}$ for a randomly selected $8 \%$ subset of reflections excluded from refinement.

As a consequence of their biological and therapeutic significance, the three-dimensional structures of about 30 chemokines have been studied to date. More recently, the structures of thymus and activation-regulated chemokine (TARC; Asojo et al., 2003) from the CC chemokines and I-TAC (Booth et al., 2004) and stroma cell-derived factor- $1 \alpha$ (SDF-1 $\alpha$; Gozansky et al., 2005) from the CXC chemokines have been determined. The structure of human IP-10 has been determined previously in three different crystal forms (Swaminathan et al., 2003). Each of these structures formed a distinct tetrameric assembly.

Glycosaminoglycans (GAGs) are linear, highly sulfated and heterogeneous polysaccharides that are often covalently linked to core proteins called proteoglycans that are present on the membrane of cells or within the extracellular matrix. They have been demonstrated to be required for the biological function of chemokines (Yu et al., 2005) and have been shown to facilitate their oligomerization (Vivès et al., 2002). Binding of chemokines to the GAG chains of cell-surface proteoglycans is thought to facilitate the formation of highly localized concentrations of chemokines, which in turn provides directional signals for leukocyte migration. Heparan sulfate has been demonstrated to be required for the presentation of chemokines on endothelial cells as well as for in vivo recruitment of leukocytes (Wang et al., 2005).

The physiological relevance of oligomerization is still not clear, but it has intrigued researchers to carry out studies to understand the mechanism of the oligomerization-induced functions of chemokines. In vivo studies with monomeric mutants of the CC chemokines RANTES (regulated upon activation, normal T-cell expressed and secreted), macrophage inflammatory protein-1 $\beta$ (MIP-1 $\beta$ ) and monocyte chemoattractant protein-1 (MCP-1) showed reduced recruitment of leukocytes (Proudfoot et al., 2003). Chemokines in monomeric forms have also been shown to be cleared more rapidly in vivo, suggesting a role of oligomerization in the localized retention of chemokines (Frevert et al., 2002). More recent studies on IP-10 mutants have clearly demonstrated the mechanism of oligomerization-dependent recruitment of activated $\mathrm{CD} 8^{+} \mathrm{T}$ cells into mice airways. The monomeric mutants were unable to induce the recruitment, although they showed significant receptor and heparin binding at higher concentrations in vitro. This suggests that the oligomerization of IP-10 is essential for its in vivo activity (Campanella et al., 2006).

Various biochemical studies and the recent discovery of its in vivo oligomerization-dependent functions prompted us to determine the three-dimensional structure of mouse IP-10. Here, we present the crystal structure of mouse IP-10 at $2.5 \AA$ resolution, which shows a novel oligomeric association. The present results provide an insight into the structural basis of oligomerization and the physiological functions of IP-10 that may contribute to further understanding of the structure and function of chemokines.

\section{Experimental procedures}

\subsection{Cloning, expression and purification}

Mouse IP-10 was cloned and expressed as described by Campanella et al. (2003) with some modifications. The recombinant plasmid was used to transform Escherichia coli BL21 (DE3) pLys strain and the culture was grown at $310 \mathrm{~K}$. Protein expression was induced with $0.2 \mathrm{~m} M$ isopropyl $\beta$-D-1thiogalactopyranoside. Cells were harvested $4 \mathrm{~h}$ after induction, pelleted and resuspended in lysis buffer containing $50 \mathrm{~m} M$ Tris- $\mathrm{HCl}$ pH 8.0 and $2 \mathrm{~m} M$ Na EDTA. Cells were lysed by sonication and the cell lysate was pelleted, resuspended and re-sonicated. $0.01 \%$ Triton $\mathrm{X}-100$ was added to the cell suspension to wash away the membrane. The pellet collected after centrifugation at $40000 \mathrm{~g}$ was dissolved in solubilization buffer containing $7 \mathrm{M}$ guanidine- $\mathrm{HCl}, 0.1 \mathrm{M}$ Tris- $\mathrm{HCl} \mathrm{pH}$ 8.0, $0.15 M$ reduced glutathione and $2 \mathrm{~m} M$ EDTA. Refolding was performed at $277 \mathrm{~K}$ following the procedure of Holloway et al. (2001). Refolded protein was diluted fivefold with MilliQ water and applied onto an SP-Sepharose column (Fast Flow, GE Healthcare) pre-equilibrated with $50 \mathrm{~m} M$ Tris- $\mathrm{HCl}$, $50 \mathrm{~m} M \mathrm{NaCl} \mathrm{pH}$ 8.0. The bound fractions were eluted using a $0.05-0.75 \mathrm{M}$ gradient of $\mathrm{NaCl}$ in $50 \mathrm{~m} M$ Tris- $\mathrm{HCl}$ buffer $\mathrm{pH}$ 8.0 at a flow rate of $1 \mathrm{ml} \mathrm{min}^{-1}$. Protein quantification was performed using the BCA assay (Pierce). Fractions containing mouse IP-10 were identified by SDS-PAGE and loaded onto a C4 RP-HPLC column pre-equilibrated with $0.1 \%$ trifluoroacetic acid in HPLC-grade water. The protein was eluted with a linear gradient of acetonitrile containing $0.08 \%$ trifluoro- 
acetic acid at a flow rate of $1 \mathrm{ml} \mathrm{min}{ }^{-1}$. The effluent was monitored at $230 \mathrm{~nm}$. Fractions containing a single band of mouse IP-10, as identified by SDS-PAGE, were pooled and lyophilized.

\subsection{Crystallization, data collection and processing}

Pure lyophilized protein was dissolved in $50 \mathrm{~m} M$ Tris- $\mathrm{HCl}$ $\mathrm{pH} 8.0$ to a concentration of $10 \mathrm{mg} \mathrm{ml}^{-1}$ and crystallization was performed by the hanging-drop vapour-diffusion method. Initial screening produced thin plates, which were optimized to improve the crystal quality. Diffraction-quality crystals were finally obtained in $0.1 M$ Tris- $\mathrm{HCl} \mathrm{pH} 8.0,0.2 \mathrm{M} \mathrm{CaCl}_{2}$ and $35 \%$ PEG 3350 after three weeks at $289 \mathrm{~K}$. A data set was collected to $2.5 \AA$ resolution at the Synchrotron Radiation Source (Station PX14.2), Daresbury, UK and was processed and scaled using the programs DENZO and SCALEPACK (Otwinowski \& Minor, 1997). The crystals belong to space group $C 2$, with unit-cell parameters $a=109.9, b=71.5$, $c=39.6 \AA, \beta=110.0^{\circ}$. The complete data statistics are given in Table 1.

\subsection{Structure determination and refinement}

The structure of mouse IP-10 was solved by maximumlikelihood molecular replacement using the program Phaser (Read, 2001) from the CCP4 program suite (Collaborative Computational Project, Number 4, 1994). The coordinates of one molecule of human IP-10 in a truncated form (residues 9-65; PDB code 107y; Swaminathan et al., 2003) were used to build the initial search model and a clear solution was obtained in space group $C 2$ with four molecules in the asymmetric unit. A stacking arrangement of molecules in the unit cell for this solution was observed in Coot (Emsley \& Cowtan, 2004), which yielded no unfavourable intermolecular contacts. Initial cycles of refinement resulted in an $R_{\text {cryst }}$ of $36.0 \%$ and an $R_{\text {free }}$ of $42.9 \%$. Iterative cycles of energy minimization, individual $B$-factor refinement and simulated annealing were

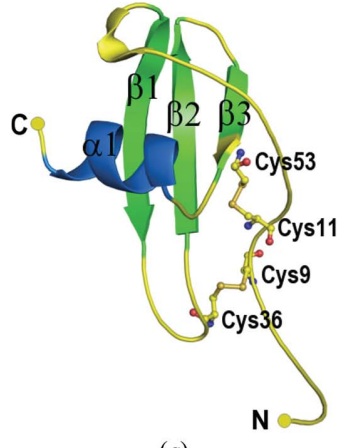

(a)

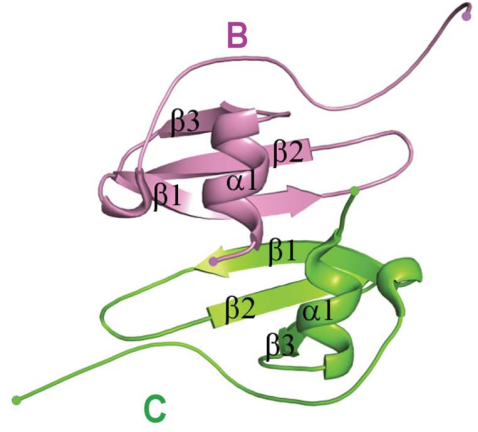

(b)
Figure 1

(a) Monomeric structure of mouse IP-10. The three $\beta$-strands and $\alpha$-helices are labelled. The $\mathrm{N}$ - and C-termini are indicated. The disulfide bonds stabilizing the N-terminal extended loop are shown in ball-andstick representation. (b) Ribbon diagram of the pseudosymmetrical dimer formed between molecules $B$ (pink) and $C$ (green), consisting of a six-stranded $\beta$-sheet with two antiparallel $\alpha$-helices. Molecules $A$ and $D$ form a similar dimer. carried out in CNS (Brünger et al., 1998), alternated with model building using Coot (Emsley \& Cowtan, 2004). Residues were replaced according to the amino-acid sequence of mouse IP-10. The positions of 81 water molecules were identified from the $\left|F_{\mathrm{o}}\right|-\left|F_{\mathrm{c}}\right|$ electron-density maps above $3 \sigma$ and were checked manually for their interactions with protein atoms. The missing residues at the $\mathrm{N}$ - and $\mathrm{C}$-termini were added as their density appeared with progressive refinement. Refinement was stopped when no further improvement in $R_{\text {free }}$ was made. The final model has an $R_{\text {cryst }}$ of $27.6 \%$ and an $R_{\text {free }}$ of $30.3 \%$. Crystallographic statistics are given in Table 1 . Figures were produced using the programs PyMOL (DeLano Scientific LLC; http://www.pymol.org) and POV-Ray (http:// www.povray.org).

\section{Results and discussion}

\subsection{Quality of the structure}

The structure of mouse IP-10 has been determined at $2.5 \AA$ resolution. The final model contains four molecules in the asymmetric unit: $A$ (residues 1-68), $B$ (residues 1-67), $C$ (residues 4-68) and $D$ (residues 4-67). Electron density for the first three $\mathrm{N}$-terminal residues could not be observed in molecules $C$ and $D$, whereas density for C-terminal residues beyond 67 (in molecules $B$ and $D$ ) and 68 (in molecules $A$ and $C$ ) could not be observed. Some disorder was observed at the C-terminal ends, thus affecting the length of the C-terminal helices. However, the C-terminal residues were not found to interact directly with heparin and CXCR3 (Campanella et al., 2003) and hence the absence of these residues did not affect our analysis. There are no breaks in the main chain and most of the side chains are located in density. Some residues at the surface show disorder or more than one conformation. The structure has good geometry, with root-mean-square (r.m.s.) deviations of $0.010 \AA$ and $1.4^{\circ}$ in bond lengths and bond angles, respectively. The Ramachandran plot (Ramachandran \& Sasisekharan, 1968) obtained using PROCHECK (Laskowski et al., 1993) showed that $77.8 \%$ of residues fall in most favoured regions, whereas $19.6 \%$ and $2.6 \%$ were in additionally and generously allowed regions, respectively. The refinement statistics are given in Table 1.

\subsection{The mouse IP-10 structure}

The structure of mouse IP-10 contains four molecules in the asymmetric unit. Each molecule exhibits the typical chemokine structural fold consisting of an extended $\mathrm{N}$-terminal loop, three antiparallel $\beta$-strands and a C-terminal helix lying obliquely across the $\beta$-sheet (Fig. 1a). All the molecules have a similar core structure, with differences in the $\mathrm{N}$ - and C-termini and loop regions. Superposition of the $\mathrm{C}^{\alpha}$ atoms of the four molecules shows an r.m.s. deviation of $0.5-1.07 \AA$. In each molecule, the $\mathrm{N}$-terminal extended loop is stabilized by two disulfide bonds between conserved cysteines (9:36 and 11:53). A dimer is formed by molecules $A$ and $D$, which are related by pseudosymmetry. Molecules $B$ and $C$ form a similar pseudosymmetrical dimer (Fig. $1 b$ ). In each of the two dimers, the $\beta 1$ 
Table 2

Hydrogen bonds between the $A-B, A-D$ and $B-C$ dimers.

Hydrogen bonds were calculated with the program HBPLUS (McDonald \& Thornton, 1994).

(a) $A-B$ interface.

\begin{tabular}{lll}
\hline Molecule $A$ & Molecule $B$ & Distance $(\AA)$ \\
\hline Val7 N & Val7 O & 2.80 \\
Val7 O & Val7 N & 2.65 \\
Arg8 N & Ala4 O & 3.11 \\
Ile14 O & Asn55 N & 2.74 \\
Asp16 O & Asn55 N & 3.14 \\
Arg38 N & Ile12 O & 3.15 \\
Asn55 N & Ile14 O & 3.09 \\
\hline
\end{tabular}

(b) $A-D / B-C$ interface.

\begin{tabular}{lll}
\hline Molecule $A(B)$ & Molecule $D(C)$ & Distance $\dagger(\AA)$ \\
\hline Lys26 N & Glu28 $\mathrm{O}^{\varepsilon 1}$ & $3.35(2.87)$ \\
Leu27 N & Ile29 O & $3.35(3.03)$ \\
Leu27 O & Ile29 N & $3.12(2.85)$ \\
$\mathrm{Glu}^{\xi} 8 \mathrm{O}^{\varepsilon 1}$ & Lys26 N & $3.42(3.27)$ \\
Ile29 N & Leu27 O & $2.92(3.05)$ \\
Ile29 O & Leu27 N & $2.99(3.10)$ \\
Lys62 N & Phe68 O & $-(3.21)$ \\
Lys66 N & Ala67 O & $-(3.25)$ \\
\hline
\end{tabular}

$\dagger$ Values in parentheses are for the $B-C$ dimer.

strands from each monomer associate to form a six-stranded $\beta$-sheet with two antiparallel $\alpha$-helices lying on one face of the $\beta$-sheet. This structural organization is similar to the dimeric CXC chemokine structures. The two dimers have an r.m.s.d of $0.59 \AA$ (122 $\mathrm{C}^{\alpha}$ atoms). The $\mathrm{C}^{\alpha}$ backbones of the pseudosymmetrical dimers in the present structure deviate by an average of $1.17 \AA$ (r.m.s.) from human IP-10 dimers (PDB codes 1o7y, 1o7z, 1o80; Swaminathan et al., 2003), while they show a $C^{\alpha}$ r.m.s. deviation of 1.4-1.6 $\AA$ compared with the dimers formed by interleukin-8 (IL-8; PDB code 3il8; Baldwin et al., 1991), platelet factor 4 (PF4; PDB code 1rhp; Zhang et al., 1994), neutrophil-activating peptide-2 (NAP-2; PDB code 1nap; Malkowski et al., 1995), growth-related oncogene- $\beta$ (Gro $\beta$; PDB code 1qnk; Qian et al., 1999) and SDF-1 $\alpha$ (PDB code 1a15; Gozansky et al., 2005) from the CXC family.
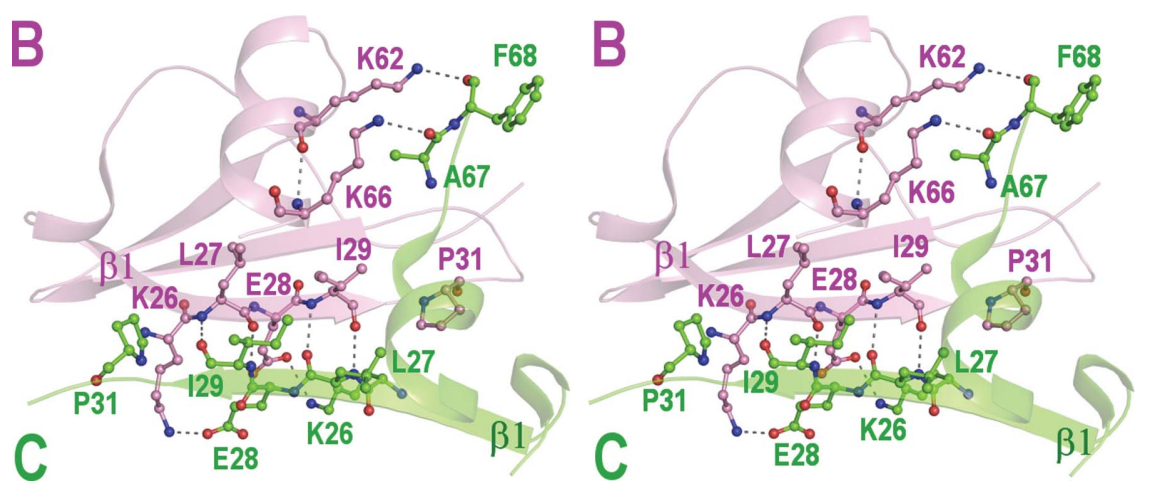

Figure 2

Stereoview of the intermolecular interactions at the $B-C$ interface. Residues from molecules $B$ and $C$ are shown in pink and green, respectively. Similar interactions between the $\beta 1$ strands were observed at the $A-D$ interface.
The dimers $(A-D$ and $B-C)$ are primarily stabilized by reciprocal interactions between opposing $\beta 1$ strands. The main-chain hydrogen bonding at the interface is limited to reciprocal interactions between residues Leu27 and Ile29 (thus accounting for four hydrogen bonds) owing to the presence of Pro31, which disrupts the $\beta$-structure. Similar interactions were observed in human IP-10 dimers owing to the conserved Pro31. However, in other CXC chemokines this position is occupied by residues other than Pro and hence four residues are involved in main-chain reciprocal interactions compared with two in the IP-10 structures. Additional interactions at the interface are contributed by salt bridges between residues Lys 26 and Glu2 8 of the $\beta 1$ strands from both the molecules in addition to extensive van der Waals contacts. The involvement of the C-terminal helices in the intermolecular interactions is limited owing to their short lengths and consists of two hydrogen bonds between residues Lys62 $\mathrm{N}^{\zeta}$ and Lys66 $\mathrm{N}^{\zeta}$ of molecule $B$ with Phe68 $\mathrm{O}$ and Ala67 $\mathrm{O}$ of molecule $C$, respectively. The ionic interactions at the C-terminal regions are absent in molecules $A$ and $D$ and only van der Waals contacts are made (Table 2, Fig. 2). The interfaces between $A$ and $D$ and between $B$ and $C$ bury 1280 and $1170 \AA^{2}$ of solvent-accessible surface area, respectively, which is comparable to the buried surface areas of other CXC chemokine dimers.

\subsection{Mouse IP-10 tetramer}

In addition to the $A-D$ and $B-C$ dimers, another dimer is formed between molecules $A$ and $B$ in the mouse IP-10 structure. This results in a distinct tetrameric assembly that is formed by the association of two pseudosymmetrical dimers: $A-D$ and $B-C$ (Fig. 3). The tetramer has an elongated structure with approximate dimensions of $90 \times 40 \AA^{2}$. Intermolecular contacts in the tetramer are through molecules $A$ and $B$. The two molecules associate through their N-terminal loops in an antiparallel fashion such that their $\alpha$-helices lie on one face of the $\beta$-strands, while the interacting $\mathrm{N}$-terminal loops are on the back of the strands. The rotation axis parallel to the $\beta$-sheets and dissecting the $A-B$ dimer relates chains $A$ and $B$ with a rotation angle of $179.1^{\circ}$, as calculated by LSQMAN (Kleywegt \& Jones, 1994). This is a novel association in which the tetramer consists of two six-stranded antiparallel $\beta$-sheets, with an antiparallel sheet formed by the $\mathrm{N}$-terminal regions between the two six-stranded $\beta$-sheets and all four helices lying on one face of the $\beta$ sheet (Fig. 4). This type of association differs significantly from many of the chemokine oligomeric structures studied to date. Intermolecular association through $\mathrm{N}$-terminal regions has been observed previously in $\mathrm{CC}$ chemokines such as MIP-1 $\beta$ (Lodi et al., 1994) and RANTES (Shaw et al., 2004). However, these dimeric structures differ considerably from the $A-B$ dimer in the 
present structure. In these $\mathrm{CC}$ chemokines, two monomers associate together such that their three-stranded $\beta$-sheets face each other with a short $\beta$-sheet formed by the interacting $\mathrm{N}$-terminal regions in the centre. The $\mathrm{C}$-terminal helix of each of the monomer lies on the exterior face of the $\beta$-sheet.

The $A-B$ dimer is primarily stabilized by the reciprocal main-chain hydrogen bonds between the two $\mathrm{N}$-terminal loops. However, the extended N-terminal loop of one molecule also interacts with the 30s loop and 50s loop of other molecule in a reciprocal manner such that a total of seven hydrogen bonds are formed at the $A-B$ interface (Fig. 4, Table 2). In addition to these hydrogen bonds, the two molecules have an extensive network of hydrophobic interactions. The solvent-accessible area at the interface in the dimer is reduced by $1770 \AA^{2}$, which is higher than the buried surface area of the pseudosymmetrical dimers $A-D$ and $B-C$ (1280 and $1170 \AA^{2}$, respectively).

The mouse IP-10 structure differs significantly in its tetrameric association from human IP-10 structures. The human IP-10 tetramer in the monoclinic space group ( $\mathrm{M}$ form) is formed by the association of two pseudosymmetrical dimers such that the two six-stranded $\beta$-sheets face each other while

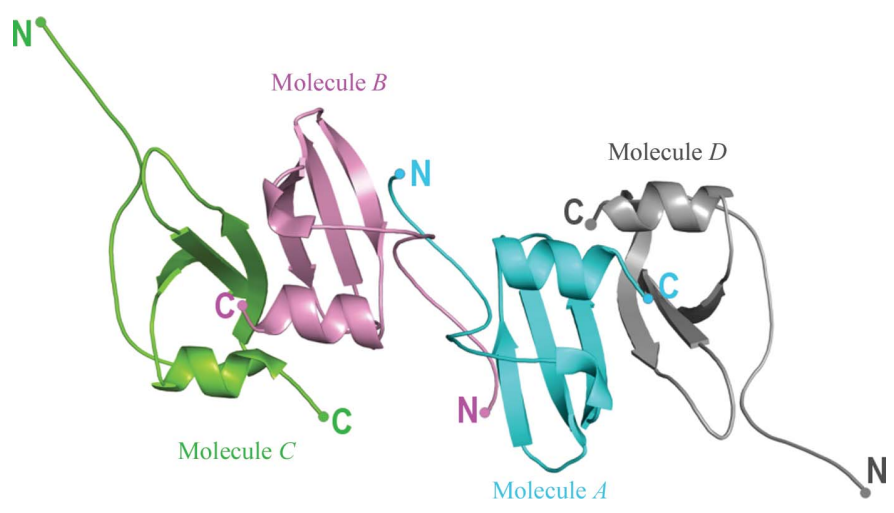

Figure 3

Schematic representation of the mouse IP-10 structure. The tetramer is formed by the association of dimers $B-C$ (shown in pink and green) and $A-D$ (shown in blue and grey).

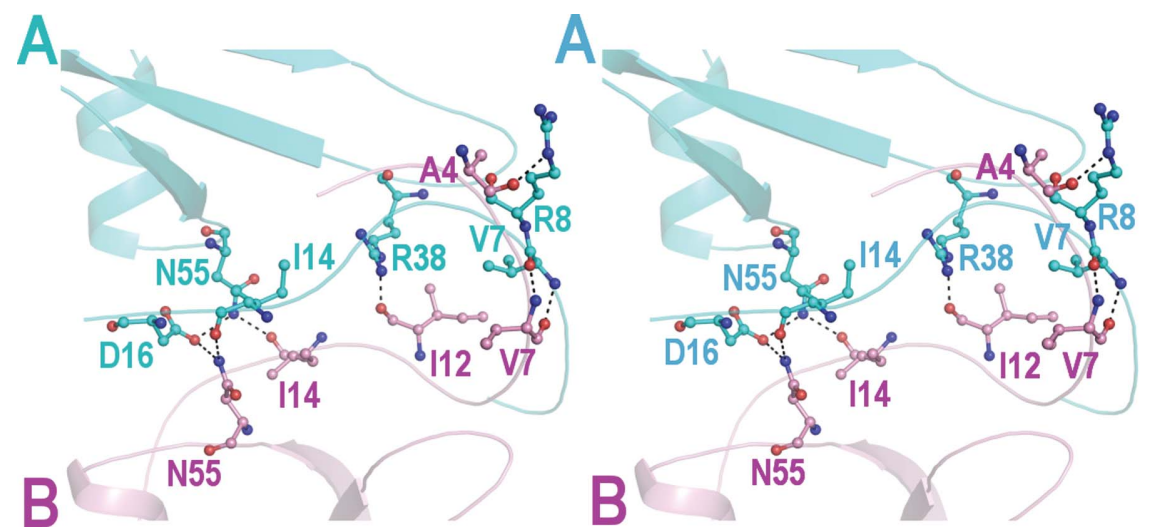

Figure 4

Stereo representation of the hydrogen bonds formed at the $A-B$ interface. Residues from molecule $A$ are shown in blue and those from molecule $B$ in pink. the C-terminal helices are present on the exterior. This arrangement of subunits is similar to the tetramers of the CXC chemokines PF4 (Zhang et al., 1994) and NAP-2 (Malkowski et al., 1995) (Fig. 5). In the tetragonal ( $\mathrm{T}$ form) and hexagonal ( $\mathrm{H}$ form) space groups, the human IP-10 dimers associate with the symmetry-related dimers through their $\beta 3$ strands to form a 12-stranded antiparallel $\beta$-sheet structure that has a sharp kink in the middle which gives an open barrel-shaped structure to the complex. However, in the H-form tetramer, the dimers also form $\mathrm{N}$-terminal asymmetric interactions, thus bringing the chains closer (Swaminathan et al., 2003). The crystal packing of the human IP-10 structures in all three space groups show different arrangements of molecules compared with the mouse IP-10 structure. In contrast to the mouse IP-10 structure, which has an elongated cylindrical shape, all these tetramers form globular-shaped structures (Fig. 5).

\subsection{Glycosaminoglycan-binding regions}

Glycosaminoglycans (GAGs) on cells bearing chemokine receptors have been reported to facilitate chemokinereceptor interactions (Hoogewerf et al., 1997). The heparinbinding residues of mouse IP-10 were determined by side-directed mutagenesis. The mutational analysis indicated residues Arg20, Arg22, Ile24, Lys26, Lys46 and Lys47 to be potentially involved in heparin binding (Campanella et al., 2003). Arg22 and Lys46 were found to be critical for binding; these residues are conserved and also form the heparinbinding site in human IP-10 (Swaminathan et al., 2003) and PF4 (Mayo et al., 1995). Single- and double-point mutations in the C-terminal helix did not affect heparin binding. However, the mutation of four basic residues at the $\mathrm{C}$-terminus to neutral and acidic residues resulted in reduced heparin binding (Campanella et al., 2003). The reduced heparin binding arising from these mutations is likely to be the consequence of an electrostatic effect (Campanella et al., 2003). The heparin-binding residues are present at the N-loop/ $\beta 1$ strand and $\beta 2$ strand/40s loop junctions in the IP-10 structure. In the pseudosymmetrical dimer $A-D$ (or $B-C$ ), these residues co-localize to form a basic cluster that lines a groove that is present on the dimeric interface along the $\beta 1$ strands. Association of the two pseudosymmetrical dimers, with each dimer having one binding site, results in the presence of two putative heparin-binding sites per tetramer. Both binding sites lie on one face of the tetramer, opposite to the helices, running along the $\beta 1$ strands and extending across the edges, thus representing maximal possible interactions with the heparin (Fig. $6 a$ ).

The potential heparin-binding residues in human IP-10 were identified as Arg22, Lys46, Lys47, Lys48, Lys62 and Lys66 (Swaminathan et al., 2003), which partially overlap with the mouse IP-10 
heparin-binding site. In the human IP-10 ( $\mathrm{M}$ form) and PF4 structures, the heparin-binding residues form a ring around the tetramers, which follows a scattered and convoluted path in the tetramers of the $\mathrm{T}$ and $\mathrm{H}$ forms of human IP-10. In all these structures, the C-terminal helices are involved in the dimeric interface such that the binding site runs perpendicular to the C-terminal helices, with residues from the loop connecting the $\mathrm{N}$-terminal region and the $\beta 1$ strand forming the extended edges. Hence, the association of the dimers might form two binding sites per tetramer, similar to those

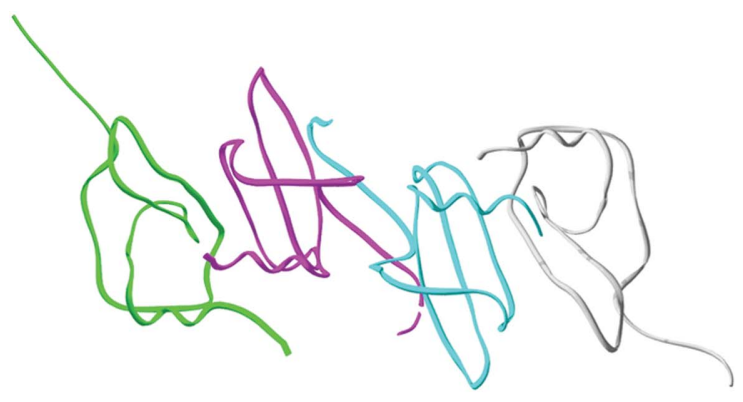

Mouse IP-10

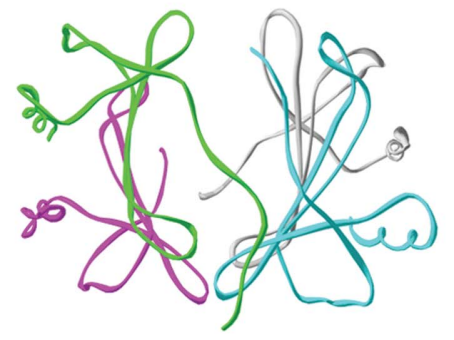

Human IP-10 $\mathrm{M}$ form

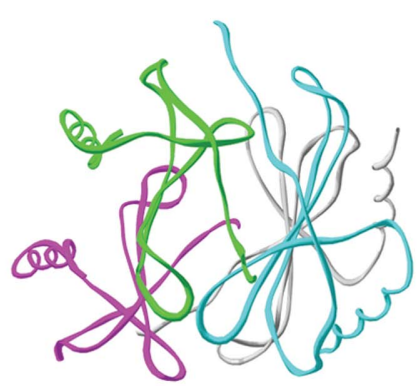

Platelet factor 4

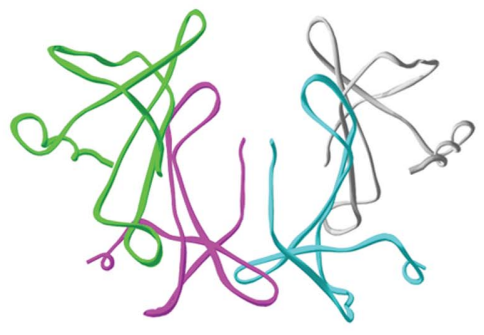

Human IP-10

$\mathrm{T}$ form

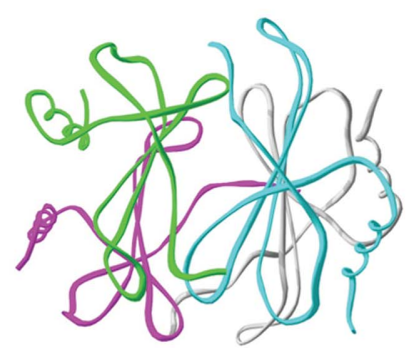

Neutrophil-activating peptide-2

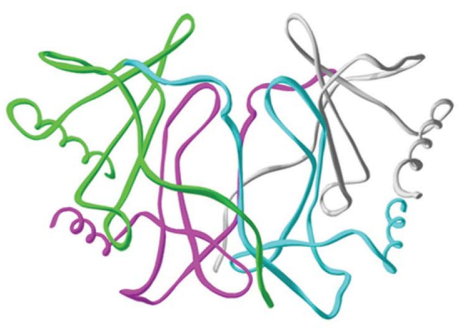

Human IP-10

$\mathrm{H}$ form

Figure 5

Backbone tracing of the oligomeric structures formed in mouse and human IP-10, platelet factor 4 and neutrophil-activating peptide-2. Four chains are shown in each structure, in which a typical chemokine dimer is formed between the green and magenta chains and the blue and grey chains, respectively. Note the different association of dimers in each structure. This figure was drawn using the program SwissPDBViewer (Guex \& Peitsch, 1997).
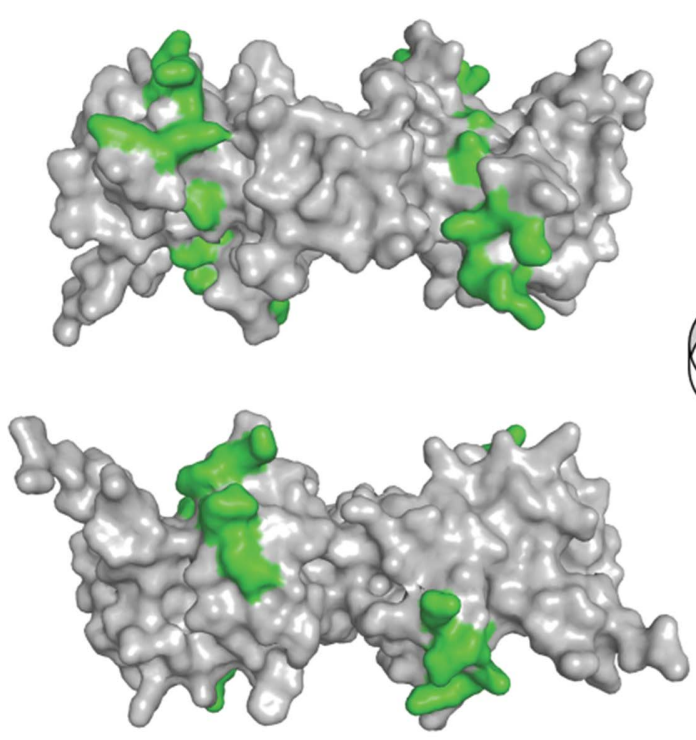

(a)
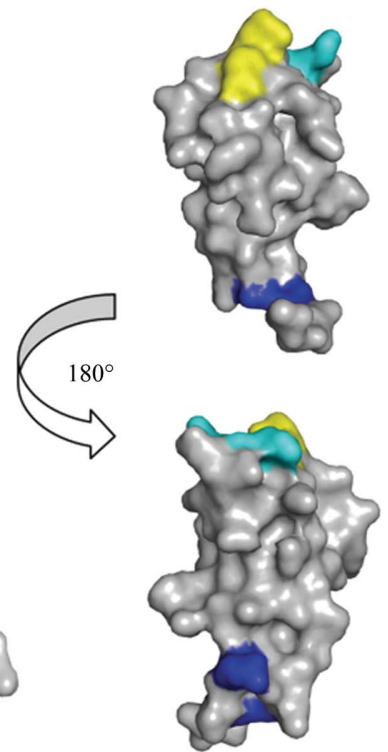

(b)

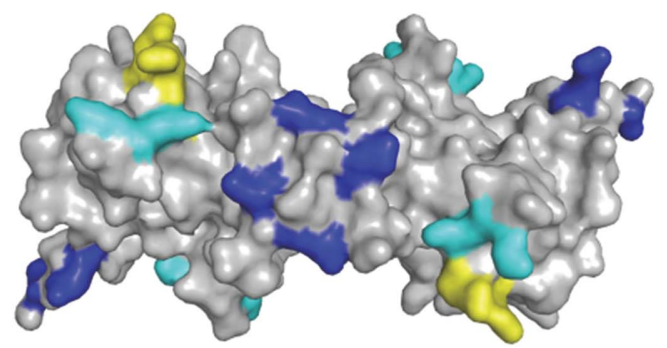

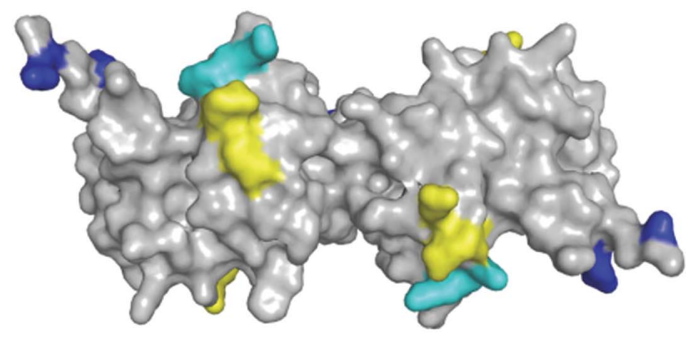

(c)

Figure 6

(a) Surface representation of the heparin-binding residues (shown in green) in the mouse IP-10 structure. The views are related by a $180^{\circ}$ rotation about the $y$ axis (the lower view is identical to that in Fig. 3). (b) The receptor-binding regions on the surface of the monomeric form of IP-10. Arg5 and Arg8 are shown in blue, Arg20, Arg22 and Ile24 in yellow and Lys46 and Lys47 in cyan. Two views are shown that are related by a rotation of $180^{\circ}$ about the $y$ axis. (c) The mapping of receptor-binding regions on the mouse IP-10 tetramer. The two views are related by a $180^{\circ}$ rotation about the $y$ axis (the lower view is the same as that in Fig. 3). 
observed in mouse IP-10. Heparin-chemokine structural modelling studies indeed showed two heparin oligosaccharides docked onto the PF4 tetramer, each oligosaccharide adopting a curved shape that runs across the pseudosymmetrical dimer perpendicular to the $\alpha$-helices and bridges the two dimers (Lortat-Jacob et al., 2002). However, the previously modelled SDF- $1 \alpha$-heparin complex shows the oligosaccharide binding to the dimer along the interface between the $\beta 1$ strands, with the $\alpha$-helices lying on the opposite face (Sadir et al., 2001). The oligosaccharide thus adopts a straight and extended shape. More recently, the crystal structure of SDF$1 \alpha$ in complex with heparin disaccharide revealed two binding sites, one of which is present at the dimer interface, thus supporting the previous studies (Murphy et al., 2007). The mouse IP-10 structure analysis indicates that the heparin oligosaccharide may bind to the IP-10 dimer in a similar fashion to that observed in SDF-1 $\alpha$. GAGs have been shown to induce chemokine oligomerization, thus forming a chemokine gradient (Proudfoot, 2006). In addition to chemokine presentation to the receptors, GAGs may play an active role in the chemokine function. For example, RANTES in complex with GAGs has been shown to have anti-HIV activity (Burns et al., 1999). Further studies are required to understand the modulation of chemokine activity by GAGs.

\subsection{CXCR3-binding regions}

IP-10-induced physiological functions are mediated by the binding of IP-10 to cell-surface CXCR3. Mutagenesis studies of mouse IP-10 identified the residues that are involved in binding to the receptor (Campanella et al., 2003). Residues in the 20s loop (Arg20, Arg22), $\beta 1$ strand (Ile24) and 40s loop (Lys46 and Lys47) were found to be important for receptor binding. The $\mathrm{N}$-terminal residues preceding the first cysteine, specifically Arg5 and Arg8, were found to be involved but not critical for receptor binding. However, Arg8 was found to be critical for CXCR3-mediated signalling. Arg8 and Lys46 are conserved in the human and mouse CXCR3 ligands. Single and double mutations in the C-terminal helix did not affect receptor binding (Campanella et al., 2003). The CXCR3binding site partially overlapped with the heparin-binding site, which further indicates that heparin may play an active role in the biological function of mouse IP-10. Fig. 6(b) shows the regions involved in receptor binding in the IP-10 monomeric structure. The receptor-binding regions are preserved when mapped onto the surface of the tetramer (Fig. $6 c$ ), indicating that the oligomerization of IP-10 may not affect its receptor binding. In human IP-10, immunological studies indicated residues 20-36 to be involved in CXCR3 binding, which also overlaps with the receptor-binding residues of mouse IP-10. Oligomerization has been demonstrated to be an essential requirement for the biological function of IP-10 (Campanella et al., 2006). Cytokine receptors have been reported to undergo dimerization upon cytokine binding (RodriguezFrade et al., 1999). The CXC chemokine SDF1- $\alpha$ has been reported to induce dimerization of the CXCR4 receptor upon binding (Vila-Coro et al., 1999). Receptor clustering is known to occur during the initiation of ligand-induced internalization in triggering the biological responses. However, receptor clustering has not yet been reported for CXCR3 molecules.

\subsection{Biological significance of oligomerization}

IP-10 has been shown to exist as higher order oligomeric forms under physiological conditions (Campanella et al., 2006). However, $N$-methylated Leu27 monomeric mutants of IP-10 had reduced heparin and CXCR3 binding but were able to induce CXCR3 internalization and chemotaxis of $\mathrm{CD} 8^{+} \mathrm{T}$ cells expressing CXCR3 at tenfold higher concentrations than wild-type IP-10 in vitro. However, the monomeric mutants failed to induce in vivo recruitment of activated $\mathrm{CD} 8^{+} \mathrm{T}$ cells. Oligomerization, rather than heparin and CXCR3 binding, was found to be essential for in vivo recruitment of $\mathrm{T}$ cells (Campanella et al., 2006). In the IP-10 structure, Leu27 is present in the $\beta 1$ strand and is involved in reciprocal mainchain hydrogen bonds with Ile29 of the other monomer forming the antiparallel dimer $A-D$ (or $B-C$ ). The presence of the $N$-methyl group disrupts this interaction and thus prevents the formation of dimers and possibly higher order complexes. Only oligomeric forms of IP-10 were able to bind to endothelial and epithelial cells in a GAG-dependent manner. The binding of oligomeric IP-10 to endothelial cells was shown to be required for the transendothelial migration of CXCR3expressing lymphocytes. The binding creates a haptotactic gradient, thus inducing the recruitment of activated $\mathrm{T}$ cells (Campanella et al., 2006). Oligomerization is therefore important for the activity of IP-10.

\section{Conclusion}

The mouse IP-10 structure presents a novel tetramer in which two typical CXC chemokine dimers associate through their $\mathrm{N}$-terminal regions to form a tetrameric assembly. Moreover, the free $\mathrm{N}$-terminal regions of two molecules at opposite ends of the tetramer increase the possibility of further association of molecules to form higher order oligomers. The presence of multiple heparin-binding sites on IP-10 oligomers might play a role in the structural stabilization of oligomers together with a role in the binding of oligomers to endothelial cells to induce the recruitment of CXCR3-expressing T cells. The cell-bound GAGs may also induce IP-10 oligomerization on the cell surface. The present study contributes to the existence of IP10 in different oligomeric forms which is important for its in vivo activity. Both mouse and human IP-10 form similar dimers, interacting though their $\beta 1$ strands, as observed for other CXC chemokines. However, the heparin- and CXCR3binding sites in human and mouse IP-10 only partially overlap. Both the structures present two heparin-binding sites per tetramer and the receptor-binding sites are preserved on the tetrameric surfaces. Investigation of the physiological significance of oligomerization is currently under way. In addition, structural studies of IP-10 in complex with GAGs will be required in order to differentiate GAG-mediated and GAGindependent oligomerization and their functional relevance. 
Since mutational studies indicated that the C-terminal helix was not directly involved in binding to both heparin and CXCR3, structural studies of IP-10 in complex with heparin and CXCR3 are required in order to establish the role of the C-terminal helix in binding and its importance in chemokine function.

We would like to acknowledge Andrew Luster and Gabriele Campanella for providing the mouse IP-10 clone and for helpful comments on the manuscript, and Michelle Hares and Daniel Holloway for their initial contribution to this project. We thank the scientists at station PX14.2 of the Synchrotron Radiation Source (Daresbury, UK), Shalini Iyer and Nethaji Thiyagarajan for their support during X-ray data collection. This work was supported by the Wellcome Trust, UK Programme Grant (No. 083191) to KRA; HJ was supported by a postgraduate studentship from the Ministry of Higher Education Malaysia and Universiti Teknologi Malaysia.

\section{References}

Asojo, O. A., Boulègue, C., Hoover, D. M., Lu, W. \& Lubkowski, J. (2003). Acta Cryst. D59, 1165-1173.

Baggiolini, M., Dewald, B. \& Moser, B. (1997). Annu. Rev. Immunol. 15, 675-705.

Baldwin, E. T., Weber, I. T., St Charles, R., Xuan, J. C., Appella, E., Yamada, M., Matsushima, K., Edwards, B. F., Clore, G. M., Gronenborn, A. M. \& Wlodawer, A. (1991). Proc. Natl Acad. Sci. USA, 88, 502-506.

Booth, V., Clark-Lewis, I. \& Sykes, B. D. (2004). Protein. Sci. 13, 2022 2028.

Brünger, A. T., Adams, P. D., Clore, G. M., DeLano, W. L., Gros, P., Grosse-Kunstleve, R. W., Jiang, J.-S., Kuszewski, J., Nilges, M., Pannu, N. S., Read, R. J., Rice, L. M., Simonson, T. \& Warren, G. L. (1998). Acta Cryst. D54, 905-921.

Burns, J. M., Lewis, J. K. \& DeVico, A. L. (1999). Proc. Natl Acad. Sci. USA, 96, 14499-14504.

Campanella, G. S., Grimm, J., Manice, L. A., Colvin, R. A., Medoff, B. D., Wojtkiewicz, G. R., Weissleder, R. \& Luster, A. D. (2006). J. Immunol. 177, 6991-6998.

Campanella, G. S., Lee, E. M., Sun, J. \& Luster, A. D. (2003). J. Biol. Chem. 278, 17066-17074.

Cole, K. E., Strick, C. A., Paradis, T. J., Ogborne, K. T., Loetscher, M., Gladue, R. P., Lin, W., Boyd, J. G., Moser, B., Wood, D. E., Sahagan, B. G. \& Neote, K. (1998). J. Exp. Med. 187, 2009-2021.

Collaborative Computational Project, Number 4 (1994). Acta Cryst. D50, 760-763.

Emsley, P. \& Cowtan, K. (2004). Acta Cryst. D60, 2126-2132.

Frevert, C. W., Goodman, R. B., Kinsella, M. G., Kajikawa, O., Ballman, K., Clark-Lewis, I., Proudfoot, A. E., Wells, T. N. \& Martin, T. R. (2002). J. Immunol. 168, 3550-3556.

Gozansky, E. K., Louis, J. M., Caffrey, M. \& Clore, G. M. (2005). J. Mol. Biol. 345, 651-658.

Guex, N. \& Peitsch, M. C. (1997). Electrophoresis, 18, 2714-2723.

Heller, E. A., Liu, E., Tager, A. M., Yuan, Q., Lin, A. Y., Ahluwalia, N., Jones, K., Koehn, S. L., Lok, V. M., Aikawa, E., Moore, K. J., Luster, A. D. \& Gerszten, R. E. (2006). Circulation, 113, 23012312.

Holloway, D. E., Hares, M. C., Shapiro, R., Subramanian, V. \& Acharya, K. R. (2001). Protein Exp. Purif. 22, 307-317.

Hoogewerf, A. J., Kuschert, G. S., Proudfoot, A. E., Borlat, F., ClarkLewis, I., Power, C. A. \& Wells, T. N. (1997). Biochemistry, 36, 13570-13578.
Kleywegt, G. J. \& Jones, T. A. (1994). CCP4/ESF-EACBM Newsl. Protein Crystallogr. 31, 9-14.

Lortat-Jacob, H., Grosdidier, A. \& Imberty, A. (2002). Proc. Natl Acad. Sci. USA, 99, 1229-1234.

Laskowski, R. A., Moss, D. S. \& Thornton, J. M. (1993). J. Mol. Biol. 231, 1049-1067.

Lodi, P. J., Garrett, D. S., Kuszewski, J., Tsang, M. L., Weatherbee, J. A., Leonard, W. J., Gronenborn, A. M. \& Clore, G. M. (1994). Science, 263, 1762-1767.

Loetscher, M., Gerber, B., Loetscher, P., Jones, S. A., Piali, L., ClarkLewis, I., Baggiolini, M. \& Moser, B. (1996). J. Exp. Med. 184, 963-969.

Loetscher, M., Loetscher, P., Brass, N., Meese, E. \& Moser, B. (1998). Eur. J. Immunol. 28, 3696-3705.

McDonald, I. K. \& Thornton, J. M. (1994). J. Mol. Biol. 238, 777793.

Malkowski, M. G., Wu, J. Y., Lazar, J. B., Johnson, P. H. \& Edwards, B. F. (1995). J. Biol. Chem. 270, 7077-7087.

Mayo, K. H., Ilyina, E., Roongta, V., Dundas, M., Joseph, J., Lai, C. K., Maione, T. \& Daly, T. J. (1995). Biochem. J. 312, 357-365.

Moser, B. \& Loetscher, P. (2001). Nature Immunol. 2, 123-128.

Murphy, J. W., Cho, Y., Sachpatzidis, A., Fan, C., Hodsdon, M. E. \& Lolis, E. (2007). J. Biol. Chem. 282, 10018-10027.

Nagpal, M. L., Davis, J. \& Lin, T. (2006). Biochem. Biophys. Acta, 1762, 811-818.

Ottonello, L. (2006). Curr. Drug Targets, 7, 1.

Otwinowski, Z. \& Minor, W. (1997). Methods Enzymol. 276, 307326.

Papadakis, K., Prehn, A. J., Zhu, D., Landers, C., Gaiennie, J., Fleshner, P. R. \& Targan, S. (2004). Inflamm. Bowel Dis. 10, 778-788.

Proudfoot, A. E. (2006). Biochem. Soc. Trans. 34, 422-426.

Proudfoot, A. E., Handel, T. M., Johnson, Z., Lau, E. K., LiWang, P., Clark-Lewis, I., Borlat, F., Wells, T. N. \& Kosco-Vilbois, H. M. (2003). Proc. Natl Acad. Sci. USA, 100, 1885-1890.

Qian, Y. Q., Johanson, K. O. \& McDevitt, P. (1999). J. Mol. Biol. 294, 1065-1072.

Qin, S., Rottman, J. B., Myers, P., Kassam, N., Weinblatt, M., Loetscher, M., Koch, A. E., Moser, B. \& Mackay, C. R. (1998). J. Clin. Invest. 101, 746-754.

Ramachandran, G. N. \& Sasisekharan, V. (1968). Adv. Protein. Chem. 23, 283-438.

Read, R. J. (2001). Acta Cryst. D57, 1373-1382.

Rodriguez-Frade, J. M., Vila-Coro, A. J., de Ana, A. M., Albar, J. P., Martinez, A. C. \& Mellado, M. (1999). Proc. Natl Acad. Sci. USA, 96, 3628-3633.

Ruschpler, P., Lorenz, P., Eichler, W., Koczan, D., Hanel, C., Scholz, R., Melzer, C., Thiesen, H. J. \& Stiehl, P. (2003). Arthritis Res. Ther. 5, R241-R252.

Sadir, R., Baleux, F., Grosdidier, A., Imberty, A. \& Lortat-Jacob, H. (2001). J. Biol. Chem. 276, 8288-8296.

Sallusto, F., Lenig, D., Mackay, C. R. \& Lanzavecchia, A. (1998). J. Exp. Med. 187, 875-883.

Shaw, J. P., Johnson, Z., Borlat, F., Zwahlen, C., Kungl, A., Roulin, K., Harrenga, A., Wells, T. N. C. \& Proudfoot, A. E. (2004). Structure, 12, 2081-2093.

Sorensen, T. L. (2004). Curr. Neurovasc. Res. 1, 183-190.

Sugiyama, K., Mukae, H., Ishii, H., Kakugawa, T., Ishimoto, H., Nakayama, S., Shirai, R., Fujii, T., Mizuta, Y. \& Kohno, S. (2006). Respirology, 11, 708-714.

Swaminathan, G. J., Holloway, D. E., Colvin, R. A., Campanella, G. K., Papageorgiou, A. C., Luster, A. D. \& Acharya, K. R. (2003). Structure, 11, 521-532.

Vila-Coro, A. J., Rodriguez-Frade, J. M., Martin De Ana, A., MorenoOrtiz, M. C., Martinez, A. C. \& Mellado, M. (1999). FASEB J. 13, 1699-1710.

Vivès, R. R., Sadir, R., Imberty, A., Rencurosi, A. \& Lortat-Jacob, H. (2002). Biochemistry, 41, 14779-14789. 
Wang, L., Fuster, M., Sriramarao, P. \& Esko, J. D. (2005). Nature Immunol. 6, 902-910.

Yu, Y., Sweeney, M. D., Saad, O. M., Crown, S. E., Hsu, A. R., Handel, T. M. \& Leary, J. A. (2005). J. Biol. Chem. 280, 3220032208 .
Zhang, X., Chen, L., Bancroft, D. P., Lai, C. K. \& Maione, T. E. (1994). Biochemistry, 33, 8361-8366.

Zhao, D., Hu, X. Y., Miller, G. G., Luster, A. D., Mitchell, R. N. \& Libby, P. (2002). J. Immunol. 169, 1556-1560.

Zlotnik, A. \& Yoshie, O. (2000). Immunity, 12, 121-127. 